\title{
Heart Rate Variability; An Index for Monitoring and Analyzing Human Autonomic Activities
}

\author{
Hiromitsu Kobayashi ${ }^{11}$, Keita Ishibashi ${ }^{11}$ and Hiroki Noguchi ${ }^{21}$
}

1) Department of Industrial Design, Kyushu Institute of Design

2) Optical Systems \& Materials R\&D Laboratory, Matsushita Electric Works, Ltd.

\begin{abstract}
A bstract. Heart rate variability (HRV), a non-invasive index of autonomic controls of the heart, has extensively been employed in various fields of study. The application range of this index persists to expand over the research horizon, and the need continues to demand technical complements for hitherto known deficiencies. Concurrent technical advances in recent years have yielded methodological techniques capable of performing quantitative analysis of HRV. However, as various approaches designed for HRV analysis exist, quantitative comparisons of the investigations have become difficult. Current physiological interpretations of the components of HRV are also inconsistent. Moreover, the issue of respiratory control has been much disputed when determining the HRV. In an attempt to clarify the current controversial state in HRV measurement, the present paper, based on the physiological background of HRV, pursued various procedures of HRV measurement and furnished global explanations for special features characterizing the various analytical approaches adopted thereof.
\end{abstract}

(Appl Human Sci, 18 (2): 53-59, 1999)

Keywords: heart rate variability, autonomic activity, respiration

\section{Introduction}

Since heart rate is the simplest physiological index, it is extensively employed for studying work-load on the living system. Heart rate on a per min basis is generally represented. In a more detailed perspective, heart rate is analyzed on a beat by beat basis (Berntson et al., 1993). In this case, the time interval of beat by beat may not be consistent and manifests a variation range of $10-30 \%$ even if the heart rate per min remains constant (Fig. 1). This phenomenon is known as heart rate variability (HRV). In addition, as heart rate is counted to practically the number of $\mathrm{R}$-wave which is the most prominent waveform of the electrocardiogram, this parameter is sometimes known as the $R-R$ interval variation. Compared to analysis of mean heart rate, information on the state of autonomic nervous system can be retrieved in greater detail.

HRV measurement is currently employed extensively in life sciences, and the application range has recently expanded to include research studies involving physiological anthropology, home economics, architecture and the other scientific fields.

Recent years have seen humans getting bored with quantitative increases in objects or environment surrounding us, and the demand for quality instead of quantity has surged. In response to this demanding trend of humans, industrial sectors have recently tuned to "comfort", and conduct product research and development with the human need as the focus.

The present paper briefly discusses the physiological background and measurement procedures of HRV for application in physiological anthropology.

\section{HRV Measurement}

Measurement of this index includes:

1. Determination of electrocardiograph (ECG)

2. Digitization of ECG

3. Detection of the R-wave peak followed by derivation of the R-R interval sequence

4. Data interpolation for securing equal interval

5. Conversion to heart rate from the reciprocal of $R-R$ interval

Items 4 and 5 are not compulsory.

The basic devices required for HRV measurement include a bio-amplifier for ECG measurement, an analog to digital ( $A D$ ) converter and a computer for R-wave analysis and data interpretation.

\section{Electrocardi ogram (ECG)}

As analyses of ECG waveforms were not the object in this HRV evaluation, practically no repercussions from the results of leading approach of ECG were encountered. For procurement of an enhanced R-wave, standard lead II 


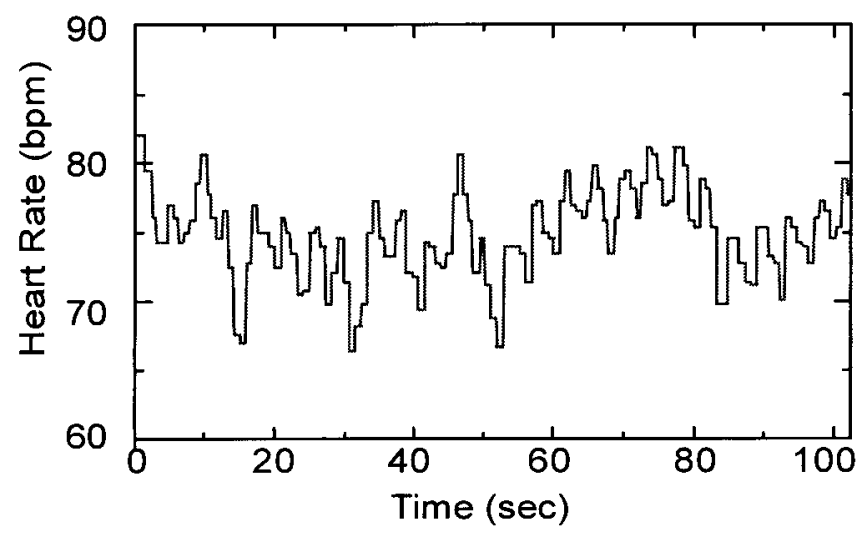

Fig. 1 Tachogram of heart rate variability. Periodic variations of heart rate are shown.

was most frequently connected on the whole. Smaller time constant was purposely appropriate to minimize artifacts from fluctuation of the baseline.

\section{A-D conversion}

It was sufficient to set the sampling frequency as 1 $\mathrm{kHz}$ in AD conversion of ECG waveforms. Through experience, we could detect the $\mathrm{R}$ wave even at $250 \mathrm{~Hz}$. However, when the sampling frequency was further reduced to a level of $100 \mathrm{~Hz}$, detection of the R-wave would then be difficult.

\section{Detection of R-wave}

To discriminate the R-wave from other components (especially the T-wave), due consideration on its special features, such as highly amplified amplitude within the shortest possible time, was taken. Supposing a certain value has been designated for the ECG waveform, and $t 1$ and $t 2$ are defined as the times when any ECG waveform crosses the fixed value from lower and upper, the apex of $\mathrm{R}$-wave is then expressed as ( $\mathrm{t} 1+\mathrm{t} 2) / 2$ (Fig. 2). This algorithm assumed the peak of waveform to lie on the center of the R-wave with left-right bilateral symmetry prevailing. As the t1-t2 interval was short, and even if artifacts resulting from waveform symmetrical displacements were produced, errors might be within the permitted range. If this is bothersome, one could adjust within the t1-t2 range until a point where the change from positive to negative gradient is observed. This detection algorithm is the most simple one, and if other restrictions are added, the consistency of detection will be further improved. Although real time processing can be done with this algorithm, construction of a noisetolerant R-wave detection system can be secured, if the real time processing are abandoned. As the R-wave amplitude is subjected to changes with the respiratory movement (Rompleman, 1987), in the R-wave amplitude-

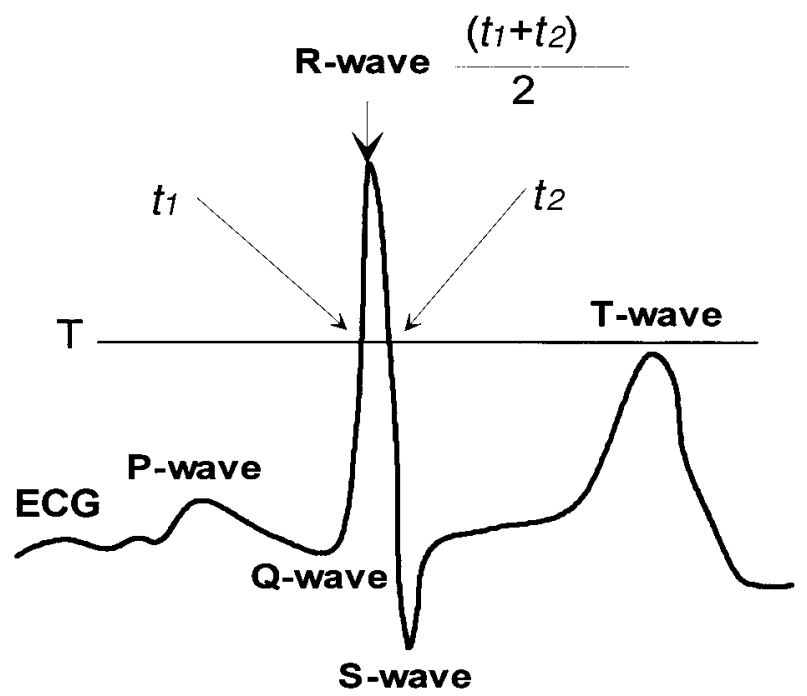

Fig. 2 Illustration of R-wave detection algorithm. t1 and t2 are the time when ECG wavefrom crosses a threshold value ( $T$ ) from lower to upper and from upper to lower, respectively. Then an apex of the $R$-wave is calculated as $(\mathrm{t} 1+\mathrm{t} 2) / 2$.

dependent detection algorithm, the source of errors is most likely to be due to changes in this R-wave amplitude rather than EMG interference. QRS detection algorithm of higher accuracy established in recent years was also introduced in Sugawara et al. (1998).

\section{Time domain analysis}

An approach in the 1960's, basically a conventional analytical approach that preceded the use of methods involving frequency analysis in this field of study, has been employed in the time domain analysis of HRV. However, the extensive use of this conventional approach, especially in the field of clinical applications, is still validated through persistent improvements.

Numerous indexes have been proposed to serve as a quantitative determinant in the time domain of HRV. Eight disparate parameters were introduced by Luczak and Laurig (1973) and 26 indexes were proposed by Opmeer (1973). In 1996, a task force set up by the European Society of Cardiology and North American Society of Pacing and Electrocardiology recommended six statistical and four geometrical indexes. Strong correlation between these indexes were the issue then. In their report, the following 4 parameters are particularly selected on a basis that the above issues are made irrelevant:

(1) SDNN (Standard deviation of all NN intervals)

(2) HRV triangular index (Total number of all NN intervals divided by the height of the histogram of all NN intervals measured on a discrete scale with bins of $7.8125 \mathrm{~ms}$ ( $1 / 128$ seconds)) 
(3) SDANN (Standard deviation of the averages of $\mathrm{NN}$ intervals in all 5-minute segments of the entire recording)

(4) RMSSD (The square root of the mean of the sum of the squares of differences between adjacent $\mathrm{NN}$ intervals)

Note that SDNN and HRV triangular index determine the global HRV; while RMSSD and SDANN represent the short and long cyclical variability, respectively.

Other than these, coefficient of variance of $R-R$ interval ( $C V$-RR $)$ has been proposed as an index for the time domain analysis. This index, originally introduced by Kageyama in 1980s, represents the standard deviations (SD) of heart beat intervals divided by the mean heart beat interval value and multiplying by a factor, 100. CV-RR basically reflects an activity of the parasympathetic nervous system, although weak responses of the sympathetic nervous system are incorporated as well.

To date, CV-RR is still extensively applied in studies related to clinical medicine and ergonomics (e.g. Hayano, 1988; Sakakibara, 1992; Hasebe 1995; Hashimoto et al., 1996).

However, frequent may be the application of this index in Japan, CV-RR is seldom employed in studies conducted by scientists outside Japan. Suzuki (1992) criticized the experimental findings based on the use of CV-RR by Kageyama et al. (1983) and raised doubts on the meaning of dividing the SD by the mean value.

Furthermore, Luczak (1979) disapproved of the trend stirred up by the proposal of indexes one after another, as quantitative comparisons of various findings yielded according to different indexes were practically unproductive then.

\section{Frequency domain analysis}

In a similar manner, numerous methods in analysis of HRV have been proposed. However, HRV is composed of complex frequency-related components, which have been known to elicit different functional aspects of the ANS. As such, HRV analyses involving procedures appropriate for interpreting frequency-related data are definitely convenient and effective.

In frequency analysis, several methods are available. The two procedures most commonly employed for HRV analysis are the fast Fourie transform (FFT) and maximum entropy method (MEM). As many publications furnish detailed explanations and interpretations on the theory and calculation approach of these methods (Akaike, 1970; Burg, 1972; Cerutti et al., 1984), our present review explores and exploits the viewpoints related to special features of the analytical methods and their applications in HRV analysis.

ME M displays characteristic features that effectively facilitate HRV analysis, and its application in biosignals has been documented in many reports related to HRV and electroencephalographic studies in recent years.

The advantages of MEM over FFT may be summarised as follows:

(1) higher spectral resolution,

(2) consistent spectra can be established with limited data,

(3) number of data is not restricted to $n^{2}$,

(4) continuous spectral values can be established.

The two problems conceivable at the same time are:

(1) the model order has to be determined by a certain method, and

(2) linearity values on spectra appropriated for steep peaks can not be guaranteed.

On the aspect of deciding the order described as the first problem, use of either the final prediction error (FPE) or Akaike information criterion (AIC) would solve the issue (Note 1). AIC or FPE can be derived by the following expression:

$$
\begin{aligned}
& \operatorname{AIC}(\mathrm{m})=\mathrm{N} \log 2 p+N \log s^{2}+N+2(\mathrm{~m}+1) \\
& \operatorname{FPE}(\mathrm{m})=\left(1+\frac{\mathrm{m}+1}{\mathrm{~N}}\right)\left(1-\frac{\mathrm{m}+1}{\mathrm{~N}}\right)^{-1} \mathrm{Em}^{2}
\end{aligned}
$$

where,

$$
E m^{2}={ }_{k=m+1}^{N}\left(y_{k}-{ }_{i=1}^{m} a_{i} y_{k-i}\right)^{2}
$$

and $\mathrm{N}$ represents the quantity of data.

The most appropriate model order then is the minimum value of the values derived thus. On an elaborative basis, AIC or FPE is derived in an ascending order, starting from the small order, and the minimum value is then derived. In doing this, the maximum value required to be computed based on these standards then becomes the issue. In general, while the order of HRV shows 3-10th under normal resting state, computations up to dimensions of 20-25th will sufficed. In the case where respiration is controlled at a constant rhythm or where high heart rate is induced by physical work-load, eventuate a order of 20th or over (Note 2).

The beat to beat interval sequence is non-equivalent interval data. By resorting to frequency analysis, an equivalent interval data can most likely be established by interpolation of the issue of data points when FFT is used. In the case of using MEM, as consistent spectra can be established even with limited data points, data can thus be interpreted without any additional interpolate procedures. This is, in fact, one of the advantages of using MEM in HRV analyses.

As for the issue of linear structural properties of the peak value, MEM spectra reflect well the alterations of frequency components, eliciting therefore no practical problems when this approach is appropriated for HRV analysis.

Although this MEM has promptly been suggested by 
Sayers (1973) for exploiting possible application in analysis of $R-R$ interval variability, its use in the analysis of $R-R$ interval variability was not realized until after 1980 . Since then, numerous findings have been documented with application of the said method (e.g., Bartolli et al., 1985; Baselli et al., 1985; Pagani et al., 1986; Kitney, 1987).

Note 1: The most appropriate orders derived by either AIC or FPE converges in most cases.

Note 2: The order derived are cases where raw data of heart rate interval series devoid of any interpolations are employed. Data with interpolations indicate higher orders.

\section{Estimation of Autonomic Activities}

\section{Two components in HRV}

Two components with different frequencies prevail in HRV: the respiratory arrhythmia (RSA) that shares the respiratory frequency and the variability related to blood pressure fluctuation characterized by a frequency approximating $0.1 \mathrm{~Hz}$ (Fig. 3). The blood-pressurerelated component is known as the Mayer-wave-related sinus arrhythmia (MWSA) or $0.1 \mathrm{~Hz}$ component

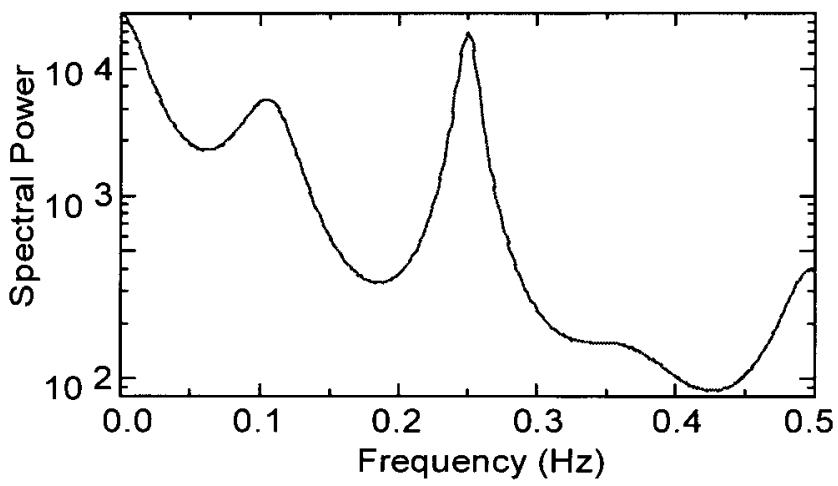

Fig. 3 HRV spectrum derived from MEM (10th order). Two components are revealed approximately in 0.1 and $0.25 \mathrm{~Hz}$. attributed to the relevant frequency displayed. The former component reflects the parasympathetic activity while the latter displays the summated sympathetic and parasympathetic activities (Pomerantz et al., 1985; Pagani et al., 1986). The attenuations of MWSA by mental work load have been reported, and numerous reports on application research have documented the substitution of this index (e.g., Hyndman and Gregory, 1975; Egelund, 1982; Vincent et al., 1987; Mulder and Mulder, 1988; Waard et al., 1995; Noguchi, 1997).

Furthermore, a component related to thermoregulatory systems (VLF) can be established at much lower frequency bands $(<0.03 \mathrm{~Hz})$ in addition to these two components (Sayers, 1973). This component appears relatively unstable and rarely poses an issue in analyses of HRV.

Pagani et al. (1986) abbreviated the low-frequency MWSA and high-frequency RSA as LF and HF, respectively (e.g., Weise et al., 1989; Ori et al., 1992; Bootsma et al., 1994; Tuininga et al., 1995; Noguchi, 1997 etc). The HRV components have since been commonly abbreviated as LF and HF. However, note that some researchers designate MWSA and RSA as MF and HF (or RF), and describe thermoregulatory component around $0.03 \mathrm{~Hz}$ as LF (e.g., Weise and Heydenreich 1989, Langewitz et al.,1991; Yeragani et al. 1994).

\section{LF and HF components}

From the power spectra of HRV, the integral power of frequency band for the respective components can be extracted and used for evaluation purposes. In such a case, the integrating frequency band becomes the focal issue. For the band widths of integration of $L F$ and $H F, a$ standard does not exist, and it may not be exaggerating to say that the situation is confusing with the procedure being controversial, complicated by the different standards adopted by the many researchers (Table 1).

With varying integration band, totally different values are produced, and direct comparisons among LF and HF values published by many scientific documents can no longer be validated.

Table 1 Frequency bands of integrating LF and HF

\begin{tabular}{lccc}
\hline & LF band (Hz) & HF band (Hz) & $\begin{array}{c}\text { Respiratory } \\
\text { control (Hz) }\end{array}$ \\
\hline Hayashi et al. (1997) & $0.05-0.15$ & $0.15-1.0$ & - \\
Malfatto et al. (1996) & $0.03-0.15$ & $0.15-0.35$ & - \\
Pagani et al. (1997) & $0.09-0.11$ & $0.22-0.24$ & - \\
Pomerantz et al. (1985) & $0.04-0.12$ & $0.224-0.280$ & 0.25 \\
Sayers (1973) & $0.07-0.10$ & - & - \\
Task force (1996) & $0.04-0.15$ & $0.15-0.40$ & - \\
Veltman and Gaillard (1998) & $0.07-0.14$ & $0.15-0.5$ & - \\
Vincente et al. (1987) & $0.06-0.14$ & - & - \\
Waard et al. (1995) & $0.07-0.14$ & - & - \\
\hline
\end{tabular}


The band-width of integration of $\mathrm{HF}$ relates to the experimental conditions where regulation of controlled respiration has indeed been imposed, or the frequency used when a controlled respiratory condition has actually been imposed. As the peak of using MWSA is most likely to occur at frequencies below $0.1 \mathrm{~Hz}$ (around 0.07-0.09 $\mathrm{Hz}$ ), it would be more precise to focus the band width of LF integration in sites slightly below $0.1 \mathrm{~Hz}$ to achieve more accurate analyses. As for the frequency band-width of $L F$, the subjects of analyses may vary according to focusing merely on MWSA or include the VLF component reflecting activities related to the body temperature regulatory mechanisms. Analyses encompassing lowfrequency components to $0.03 \mathrm{~Hz}$ in the band width of LF integration would contain the latter choice (e.g., Malfatto et al., 1996).

\section{HRV components and autonomic activities}

Current interpretations using $\mathrm{HF}$ as an index for the parasympathetic nervous system encounters little or no dispute among the various scientists. However, varying interpretations on the analysis are attempted when LF is used. The LF analysis may be interpreted as follows:

1. LF is considered as an index for both sympathetic and parasympathetic activities.

2. LF is considered as an index for sympathetic activity.

Based on the experimental findings by Pomerantz et al. (1985), LF is often considered as an index for both sympathetic and parasympathetic responses. However, if the findings of Pomerantz et al. were to be adopted, the component enveloping the sympathetic components containing the LF would compose $17 \%$ of the total, revealing major involvement of the parasympathetic components. In other experimental results, the contribution of sympathetic components are of an ever lesser rate (e.g., Langewitz et al., 1991; Grossman and Kollai, 1993; Cacioppo et al., 1994).

The idea that LF reflects sympathetic activity is based upon the fact that LF may be in relation to the blood pressure fluctuation and the fact that LF shows significant increase during orthostatics (Weise and Heydenreich, 1989; Kobayashi, 1996).

From pharmacological studies using various blockers, LF may not be the only index representing sympathetic activities. The fact that LF increases in orthostatic load and its correlation with mean heart rate implies that it may be difficult to expound phenomena where LF is used as the index reflecting sympathetic activities.

Furthermore, explanations of the relevant $\mathrm{LF} / \mathrm{HF}$ ratio may be pursued as follows:

1. The LF/HF ratio as an index balancing the sympathetic and parasympathetic responses, and

2. The LF/HF is considered as an index of sympathetic activities.
The interpretation with the LF/HF ratio as an index of sympathetic activities per se, LF is then considered as an index of both sympathetic and parasympathetic responses, where the dividing factor HF will render extraction of only the sympathetic effects possible. However, simply dividing by HF may not be able to completely eradicate the parasympathetic components from LF. As such, the LF/HF ratio may therefore appropriately interpret and serve well as an index equilibrating the sympathetic and parasympathetic activities. In strict sense, the implication of this "equilibrium" does not represent the ratio of sympathetic/ parasympathetic activity, and it cannot be denied that this approach represents a mere approximation.

Much room remains for discussion on the indexautonomic activity relationship, and future research studies will definitely complement the imperfect and incomplete status on indexing the state of the autonomic nervous system.

\section{Control of respiration}

The respiratory frequency approximates to $0.2-0.4$ $\mathrm{Hz}$ during resting in general. In this case, although it is possible to separate the frequency band between MWSA and RSA, the respiratory frequency may vary greatly because of various factors, leading often to overlapping of band-widths of the LF component (Sayers,1973; Kobayashi, 1995). As a result, clear separation between MWSA and RSA cannot be realized. As low-frequency respiration in the vicinity of $0.1 \mathrm{~Hz}$ displays extremely high amplitudes (Angelone and Coulter, 1964; Hirsch and Bishop, 1981; Kobayashi, 1997), values of the LF component play an influential role. This may be one of the factors contributing to HRV inconsistencies (Ori et al., 1992; Kobayashi et al., 1995).

From the above argument, it is therefore essential to impose control in conditioning a constant respiratory rhythm by using a metronome and other corrective procedures in measuring HRV. In fact, measurements of the HRV are often performed with regulatory control imposed on the subject to secure a constant respiratory frequency in recent times (e.g., Mukae and Sato, 1992; Puig et al., 1993). Respiratory frequency is not the only factor of concern in HRV; the tidal volume has to be considered as well. As such, in a stricter sense, controls of the respiratory frequency and tidal volume have to be regulated (Kobayashi, 1998).

\section{References}

Akaike H (1970) Statistical predictor identification. Ann Inst Ststist Math 22: 203-217

Angelone A, Coulter NA (1964) Respiratory sinus arrhythmia: a frequency dependent phenomenon. J Appl Physiol 19: 479-482 
Bartolli F, Baselli G, Cerutti S (1985) AR identification and spectral estimate applied the $R-R$ interval measurement. Int J Bio-medical Computing 16: 201215

Baselli G, Bolis D, Cerutti S, Freschi C (1985) Autoregressive modeling and power spectral estimate of $R-R$ interval time series in arrhythmic patients. Computers and Biomedical Research 18: 510-530

Berntson GG, Cacioppo J T, Quigley KS ( 1993) Respiratory sinus arrhythmia: Autonomic origins, physiological mechanisms, and psychophysiological implications. Psychophysiology 30: 183-196

B ootsma M, Swenne CA, Bolhuis HHV, Chang PC, Cats VM, Bruschke AVG (1994) Heart rate and heart rate variability as indexes of sympathvagal balance. Am J Physiol 266: H1565-1571

Burg JP (1972) The relationship between maximum entropy spectra and maximum likelihood spectra. Geophysics 37: 375-376

Cacioppo JT, Berntson GG, Binkley PF, Quigley KS, Uchino BN, Fieldstone A (1994) Autonomic cardiac control II. Noninvasive indices and basal response as revealed by autonomic blockades. Psychophysiology 31: $586-598$

Cerutti S, Baselli G, Liberati D (1984) Autoregressive filtering in heart rate variability signal. In Costatinides CV ed. Digital signal processing-84. North-Holland, A msterdam

Egelund N (1982) Spectral analysis of heart rate variability as an indicator of driver fatigue. Ergonomics 25 (7): 663-672

Grossman P, Kollai M (1993) Respiratory sinus arrhythmia, cardiac vagal tone, and respiration: Withinand between-indivisual relations. Psychophysiology 30: 486-495

Hasebe Y, Iriki M, Takahashi K (1995) Usefulness of $r$ $r$ interval and its variability in evaluation of thermal comfort. Int J Biometeorol 38: 116-121

Hashimoto A, Maeda H, Yokoyama M (1996) Augmentation of parasympathetic nerve function in patients with extrinsic bronchial asthma- evaluation by coefficiency of variance of $R-R$ interval with modified long-term ECG monitoring system. Kobe J Med Sci 42: 347-359

Hayano J (1988) Quantitative assessment of autonomic function by autoregressive spectral analysis of heart rate variability: $E$ ffect of posture, respiratory frequency, and age. J Autonomic Nerv Sys 25 (3): 334-344

Hayashi N, Nakamura Y, Muraoka I (1997) Little effect of endurance training on heart rate and heart rate variability at sitting rest. Adv Exerc Sports Physiol 3 (1) : 17-22

Hirsch J A, Bishop B (1981) Respiratory sinus arrhythmia in humans: how breathing pattern modulates heart rate. Am J Physiol 10: H620-H629

Hyndman BW, Gregory J R (1975) Spectral analysis of sinus arrhythmia during mental loading. Ergonomics 18 (3) : $255-270$

Kageyama et al. (1983) A clinical label of diabetic autonomic neuropathy. Tohoku J Exp Med 141: 479483

Kitney RI ( 1987) Beat by beat inter-relationships between heart rate, blood pressure, and respiration. In Kitney $\mathrm{RI}$, Rompelman $\mathrm{O}$ eds. The beat by beat investigation of cardiovascular function. Clarendon Press, Oxford: 146-178

Kobayashi H (1996) Postural effect on respiratory sinus arrhythmia with various frequencies. Appl Hum Sci 15 (2): 87-91

Kobayashi H (1997) F requency responses of respiratory sinus arrhythmia. Jap J Physiol Antrop 2 (2): 7-12 (in J apanese with English abstract)

Kobayashi H (1998) Normalization of respiratory sinus arrhythmia by factoring in tidal volume. Appl Hum Sci 17 (5): 207-213

Kobayashi H, Mukae H, Machida T (1995) Heart rate variability and mental work load. Design of amenity. Selected papers of international symposium, Kyushu Univ Press: 259-265

Langewitz W, Rüddel $H$, Schächinger $\mathrm{H}$, Lepper W, Mulder LJ M, Veldman JZHP, Roon AV (1991) Changes in sympathetic and parasympathetic cardiac activation during mental load: An assessment by spectral analysis of heart rate variability. Homeostasis 33: 23-33

Luczak H (1979) Fractioned heart rate variability. Part II: Experiments on superimposition of component stress. Ergonomics 22 (12): 1315-1323

Luczak H, Laurig W (1973) An analysis of Heart rate variability. Ergonomics 16 ( 1): 85-97

Malfatto G, Facchini M, Bragato R, Branzi G, Sala L, Leonetti G (1996) Short and long term effects of exercise training on the tonic autonomic modulation of heart rate variability after myocardial infraction. Eur Heart J 17: 532-538

Mukae H, Sato M (1992) The effect of color temperature of lighting sources on the autonomic nervous functions. Ann Physiol Anthrop 11 (5): 533-538

Mulder LJ M, Mulder G (1988) Cardiovascular reactivity and mental work-load. In Kitney RI, Rompelman O, eds. The beat by beat investigation of cardiovascular function. Clarendon Press, Oxford: 216-253

Noguchi Y (1997) Vehicle evaluation by measuring physiological reactions: using the HRV index. Heavy Veh Syst 4 (2/4): 323-339

Opmeer CHJM (1973) The information content of successive R-R interval times in ECG. Preliminary results using factor analysis and frequency analysis. Ergonomics 16 (1): 105-112

Ori Z; Monir G, Weiss J, Sayhouni X, Singer DH (1992) Heart rate variability. Frequency domain analysis. Cardiol Clin 10: 499-537 
Pagani M, Lombardi F, Guzzetti S, Rimoldi O, Furlen R, Pizzinelli P, Sandrone G, Malfatto G, 'Orto GD, Piccaluga E, Turiel M., Basello G, Cerutti S, Malliani A (1986) Power spectral analysis of heart rate and arterial pressure variabilities as a maker of sympathovagal interaction in man and conscious dog. Circulation Research 59 (2): 171-192

Pagani M, Mantano N, Porta A, Malliani A, Abboud F M, Birkett C, Sommers VK (1997) Relationship between spectral component of cardiovascular variabilities and direct measures of muscle sympathetic nerve activity in humans. Circulation 95: 1441-1448

Pomeranz B, Macauly RJ B, Caudill MA, Kutz I, Adam $D$, Gordon D, Kilborn KM, Barger AC, Shannon DC, Cohen RJ, Benson H ( 1985) Assessment of autonomic function in humans by heart rate spectral analysis. Am J Physiol 248: 151-153

Puig J, Fareitas J, Carvalho MJ, Puga N, Ramos J, Fernandes P, Costa O, Freitas AFD (1993) Spectral analysis of heart rate variability in athletes. J Sports Med Phys. Fitness 33: 44-48

Rompelman O (1987) Accuracy aspects in ECGpreprocessing for the study of heart-rate variability. In Kitney RI, Rompelman 0 eds. The beat by beat investigation of cardiovascular function. Clarendon Press, Oxford, 103-125

Sakakibara M (1992) Assessment of autonomic function by the spectral analysis of heart rate variability: an examination in a mirror drawing task. Shinrigaku Kenkyu 63: 123-127

Sayers B MCA ( 1973) Analysis of heart rate variability. Ergonomics 16 (1): 17-32

Sugawara A, Matsumoto M, Suzuki M, Dohi T (1998) Morphological QRS-complex detection for a vital and life information mailing system. BME 36 (1): 32-40 (in Japanese with English abstract)

Suzuki Y (1992) Study on sinus rhythm variational indices as a functional test of autonomic dysfunction in diabetic neuropathy. Tokyo J osiikadaigaku Zassi 62 (3) : 224250
Task Force of the European Society of Cardiology and the North American Society of Pacing and Electrophysiology (1996) Heart Rate Variability Standards of Measurement, Physiological Interpretation, and Clinical Use Circulation 93: 1043-1065

Tuininga YS, Crijns HJ GM, Brouwer J, Berg MP, Veld AJ M, Mulder G, Lie KI ( 1995) Evaluation of importance of central effects of atenolol and metprolol measured by heart rate variability during mental performance tasks, physical exercise, and daily life in stable postinfract patients. Circulation 92: 3415-3423

Veltman JA, Gaillard AWK (1998) Physiological workload reactions to increaseing levels of task difficulty. Ergonomics 41 (5): 656-669

Vicente KJ, Thornton DC, Moray N (1987) Spectral analysis of sinus arrhythmia: A measure of mental effort. Human Factors 29 (2): 171-182

Waard D, J essurun M, Steyvers FJ J M (1995) Effect of road layout and road environment on driving performance, driver's physiology and road appreciation. Ergonomics 38 (7): 1395-1407

Weise F, Baltrusch K, Heydenreich F (1989) Effect of low-dose atropine on heart rate fluctuations during orthostatic load: a spectral analysis. J Autonomic Nerv Sys 26: 223-230

Weise F, Heydenreich F (1989) Effect of modified respiratory rhythm on heart rate variability during active orthostatic load. Biomed Biochem Acta 48 (8): 549-556

Yeragani VK, Pohl R, Berger R, Balon R, Srinivasan K (1994): Relationship between age and heart rate variability in supine and standing postures: a study of spectral analysis of heart rate. Pediatric Cardiology 15 (1) : $14-20$

Received: J anuary 8, 1999

Accepted: February 13, 1999

Correspondence to: Hiromitsu Kobayashi, Department of Industrial Design, Kyushu Institute of Design, 4-91 Shiobara, Minami-ku, Fukuoka 815-8540, Japan 